\title{
PERFIL EPIDEMIOLÓGICO DO TRAUMA DE FACE DOS PACIENTES ATENDIDOS NO PRONTO SOCORRO DE UM HOSPITAL PÚBLICO
}

\author{
EPIDEMIOLOGY OF FACIAL TRAUMA OF PATIENTS ADMITTED TO A PUBLIC \\ HOSPITAL EMERGENCY DEPARTMENT
}

\author{
Jefferson Lessa Soares de Macedo TCBC-DF ${ }^{1}$ : Larissa Macedo de Camargo ${ }^{2}$; \\ Pedro Fragoso de Almeida ${ }^{2}$; Simone Corrêa Rosa ${ }^{3}$
}

\begin{abstract}
RESUMO: Objetivo: Avaliar os dados epidemiológicos e a localização dos traumas de face de pacientes atendidos no Hospital Regional da Asa Norte (HRAN), Brasília, Distrito Federal. Método: Trata-se de um estudo retrospectivo, realizado no pronto socorro do HRANDF, visando avaliar o perfil epidemiológico dos pacientes atendidos pela equipe da Unidade de Cirurgia Plástica vítimas de trauma de face no período de 1 de janeiro a 31 dezembro de 2004. Resultados: O estudo compreendeu 711 pacientes, destacando-se o sexo masculino $(72,8 \%)$. Quanto à causa, predominou a agressão física, seguida por acidente com veículos/motos. As quedas foram a causa predominante das lesões em crianças, mas verificou-se a participação cada vez maior da agressão física como mecanismo de trauma facial com o aumento da idade. A relação de homem:mulher foi de 3:1. A faixa etária mais atingida foi de 21 a 30 anos, representando $35,3 \%$ dos pacientes. As fraturas foram encontradas em $24,9 \%$ das lesões faciais. O nariz foi o local mais acometido nas fraturas de face (76,8\%). Conclusão: A violência interpessoal foi a principal causa de trauma de face. A queda da própria altura mostrou-se como importante mecanismo de trauma nos extremos de idade (Rev. Col. Bras. Cir. 2008; 35(1): 009-013).
\end{abstract}

Descritores: Face; Traumatismos maxilofaciais; Ossos da face; Traumatismos faciais; Ferimentos e lesões; Violência; Cirurgia.

\section{INTRODUÇÃO}

Os traumas apresentam grande importância na sociedade contemporânea. Segundo dados da Organização Mundial de Saúde, os traumas estão entre as principais causas de morte e morbidade no mundo. A cada dia, 16.000 pessoas morrem em decorrência de trauma ${ }^{1}$. Dentre os diversos tipos, o trauma de face destaca-se pela sua importância, uma vez que apresenta repercussões emocionais, funcionais e possibilidade de deformidades permanentes ${ }^{2,3}$. Além disso, representa 7,4\%-8,7\% dos atendimentos efetuados na emergên$\operatorname{cia}^{4,5}$.

A grande quantidade de lesões na face deve-se à enorme exposição e à pouca proteção desta região o que acarreta freqüentemente lesões graves. As lesões da cabeça e da face podem representar $50 \%$ de todas as mortes traumáticas ${ }^{6}$.

Trata-se de um trauma de abrangência multidisciplinar, envolvendo principalmente as especialidades de cirurgia geral, oftalmologia, cirurgia plástica, bucomaxilofacial e neurocirurgia ${ }^{7}$.

Ressalta-se que o procedimento cirúrgico precoce no trauma de face oferece os melhores resultados, além de contribuir para promover a volta do paciente, mais rapidamente, às atividades profissionais e ao convívio da família ${ }^{8}$.
Este estudo tem por objetivo analisar os dados epidemiológicos dos pacientes atendidos no Serviço de Cirurgia Plástica do Hospital Regional da Asa Norte, Brasília, Distrito Federal no ano de 2004.

\section{MÉTODO}

Trata-se de um estudo retrospectivo, sendo realizado através da análise de prontuários e das guias de atendimento de emergência (GAEs) dos pacientes atendidos na Unidade de Emergência do Serviço de Cirurgia Plástica do Hospital Regional da Asa Norte (HRAN), Brasília, Distrito Federal, no período de janeiro a dezembro de 2004.

Todos os pacientes atendidos no pronto socorro do HRAN pela equipe da Cirurgia Plástica, vítimas de trauma na face no ano de 2004, foram incluídos. Os dados foram coletados utilizando uma ficha que incluía as seguintes variáveis: data e horário do atendimento e do trauma, idade, gênero, procedência, causa do acidente, características das lesões, lesões associadas, tipo de anestesia e tratamento.

Os casos foram classificados por etiologia em sete categorias: agressão física (incluíram violência interpessoal e mordeduras), atropelamento (incluíram carro, caminhão, motocicleta, bicicleta e ônibus), acidente com veículos (incluí-

1. Cirurgião Plástico do Serviço de Cirurgia Plástica do Hospital Regional da Asa Norte; Mestre e Doutor em Medicina pela Universidade de Brasília; Docente de Medicina da Escola Superior de Ciências da Saúde (ESCS), Fundação de Ensino e Pesquisa em Ciências da Saúde (FEPECS), Secretaria de Saúde do Distrito Federal (SESDF), Brasília - DF

2. Acadêmico de Medicina da ESCS, FEPECS, SES, Brasília - DF; Bolsistas do CNPq

3. Cirurgiã Plástica do Serviço de Cirurgia Plástica do Hospital Regional da Asa Norte; Mestre em Medicina pela Universidade de Brasília.

Recebido em 30/08/2007

Aceito para publicação em 25/10/2007

Conflito de interesses: nenhum

Fonte de financiamento: nenhuma

Trabalho realizado na Unidade de Emergência do Serviço de Cirurgia Plástica do Hospital Regional da Asa Norte - HRAN, Secretaria de Saúde do Distrito Federal, Brasília - DF. 
ram colisão contra outros veículos, postes, paredes, casas e queda de motocicleta), esporte (incluíram várias modalidades esportivas, inclusive lutas marciais), acidente de bicicleta, queda da própria altura e impacto não relacionado à queda, nesta última categoria foram incluídos traumas de colisão direta com móveis, instalações, elementos internos e externos das residências dos pacientes.

Os dados foram analisados e codificados pelo Programa Epi Info, versão 3.2.2. O projeto foi aprovado pelo Comitê de Ética em Pesquisa da Secretaria de Estado de Saúde do Distrito Federal.

\section{RESULTADOS}

O estudo compreendeu 711 pacientes, destacandose o sexo masculino, representando $72,8 \%$ dos pacientes. A faixa etária de maior incidência de trauma facial foi de 21 a 30 anos, compreendendo $35,3 \%$ de toda a população estudada (Figura 1).

A média de idade foi de 27 anos (variação de 1 a 84 anos). Quanto à procedência, $82 \%$ residiam no Distrito Federal, 16,3\% em Goiás e 1,7\% em outros Estados.

$\mathrm{O}$ atendimento foi realizado principalmente nas primeiras 6 h após o trauma, correspondendo a $47,5 \%$ dos pacientes. Quanto ao período de maior demanda no pronto-socor- ro, destaca-se o período da tarde (das $12 \mathrm{~h}$ às $17 \mathrm{~h} 59 \mathrm{~min}$ ), seguido pelo período da manhã (das $6 \mathrm{~h}$ às $11 \mathrm{~h} 59 \mathrm{~min}$ ) e da noite, respectivamente.

Quanto à causa dos traumas faciais, predomina a agressão física, em ambos os sexos, correspondendo a 38,8\% das vítimas de trauma de face. Com relação ao sexo masculino, além da agressão física, ressaltam-se os acidentes com veículos/motos responsáveis por $14,9 \%$ dos traumas de face. Por outro lado, no sexo feminino, a segunda causa de agravo foi a queda da própria altura, correspondendo a $29 \%$ dos traumas em mulheres (Tabela 1).

Avaliando-se a natureza dos traumas faciais relacionados à faixa etária, verificou-se que $42,7 \%$ das crianças (faixa etária entre 0 e 10 anos) sofreram queda da própria altura. Nas faixas etárias compreendidas entre os 11 e 50 anos, agressão física foi a principal causa dos traumas de face. Os pacientes, com idade superior a 60 anos, tiveram como principal causa, a queda da própria altura, correspondendo a $73,4 \%$ dos casos (Tabela 2).

Quanto ao sítio das lesões, a região zigomática foi a principal região afetada compreendendo a 36\%, seguida pela região nasal que representou $24,7 \%$ das regiões acometidas (Tabela 3). Quanto às características das lesões, e sabendo que se trata de um trauma com múltiplas lesões, as contusões/ escoriações prevaleceram como o tipo de lesão mais verificada,

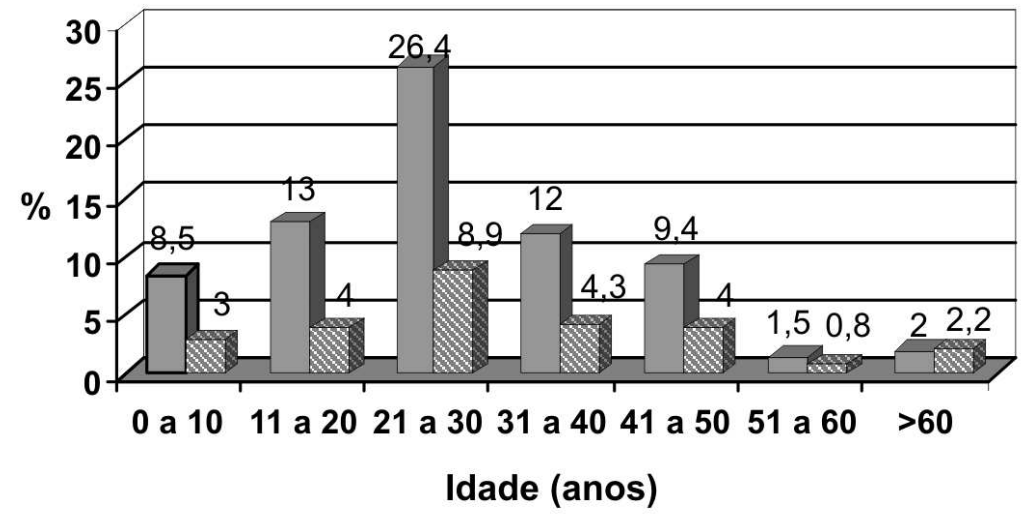

Masculino

Feminino

Figura 1 - Pacientes com trauma facial, segundo sexo e idade (anos).

Tabela 1 - Pacientes com trauma facial, segundo etiologia e sexo.

\begin{tabular}{|c|c|c|c|c|c|c|}
\hline \multirow[t]{3}{*}{ Etiologia } & \multicolumn{6}{|c|}{ Sexo } \\
\hline & \multicolumn{2}{|c|}{ Masculino } & \multicolumn{2}{|c|}{ Feminino } & \multicolumn{2}{|c|}{ Total } \\
\hline & $\mathbf{N}$ & $\%$ & $\mathbf{N}$ & $\%$ & $\mathbf{N}$ & $\%$ \\
\hline Acidente veículo/moto & 77 & 14,9 & 33 & 17,1 & 110 & 15,5 \\
\hline Agressão física & 202 & 39,0 & 74 & 38,4 & 276 & 38,8 \\
\hline Atropelamento & 7 & 1,4 & 3 & 1,6 & 10 & 1,4 \\
\hline Esporte & 50 & 9,7 & 2 & 1,0 & 52 & 7,3 \\
\hline Impacto não relacionada a queda & 62 & 12,0 & 17 & 8,8 & 79 & 11,2 \\
\hline Queda da própria altura & 51 & 9,8 & 56 & 29,0 & 107 & 15,0 \\
\hline Queda de bicicleta & 69 & 13,2 & 8 & 4,1 & 77 & 10,8 \\
\hline Total & 518 & 100 & 193 & 100 & 711 & 100 \\
\hline
\end{tabular}


Tabela 2 - Pacientes com trauma facial, segundo causa e idade.

\begin{tabular}{|c|c|c|c|c|c|c|c|c|c|c|c|c|c|c|}
\hline \multirow{3}{*}{ Causas } & \multicolumn{12}{|c|}{ Idade (anos) } & & \\
\hline & \multicolumn{2}{|c|}{0 a 10} & \multicolumn{2}{|c|}{11 a 20} & \multicolumn{2}{|c|}{21 a 30} & \multicolumn{2}{|c|}{31 a 40} & \multicolumn{2}{|c|}{41 a 50} & \multicolumn{2}{|c|}{51 a 60} & \multicolumn{2}{|c|}{$>60$} \\
\hline & $\mathbf{N}$ & $\%$ & $\mathbf{N}$ & $\%$ & $\mathbf{N}$ & $\%$ & $\mathbf{N}$ & $\%$ & $\mathbf{N}$ & $\%$ & $\mathbf{N}$ & $\%$ & $\mathbf{N}$ & $\%$ \\
\hline Acidente veículo/moto & 1 & 1,2 & 17 & 14 & 53 & 21 & 18 & 15,5 & 18 & 18,9 & 2 & 11,8 & 1 & 3,3 \\
\hline Agressão física & 14 & 17,1 & 61 & 50,4 & 104 & 42 & 56 & 48,2 & 36 & 38 & 3 & 17,6 & 2 & 6,7 \\
\hline Queda de bicicleta & 16 & 19,5 & 16 & 13,2 & 28 & 11 & 6 & 5,2 & 10 & 10,5 & 0 & 0 & 1 & 3,3 \\
\hline Esporte & 0 & 0 & 15 & 12,4 & 29 & 12 & 6 & 5,2 & 2 & 2,1 & 0 & 0 & 0 & 0 \\
\hline Impacto não relacionado à queda & 14 & 17,1 & 7 & 5,8 & 17 & 6,8 & 18 & 15,5 & 16 & 16,8 & 4 & 23,5 & 3 & 10 \\
\hline Queda da própria altura & 35 & 42,7 & 5 & 4,2 & 17 & 6,8 & 11 & 9,5 & 11 & 11,6 & 6 & 35,3 & 22 & 73,4 \\
\hline Atropelamento & 2 & 2,4 & 0 & 0 & 2 & 0,8 & 1 & 0,9 & 2 & 2,1 & 2 & 11,8 & 1 & 3,3 \\
\hline Total & 82 & 100 & 121 & 100 & 250 & 100 & 116 & 100 & 95 & 100 & 17 & 100 & 30 & 100 \\
\hline
\end{tabular}

Tabela 3 - Pacientes com trauma facial - localização da lesão.

\begin{tabular}{lrc}
\hline Localização da lesão & N & \% \\
\hline Auricular & 40 & 5,6 \\
Couro cabeludo & 45 & 6,4 \\
Frontal & 36 & 5,1 \\
Labial & 22 & 3,2 \\
Mandibular & 33 & 4,7 \\
Mentoniana & 20 & 2,4 \\
Nasal & 175 & 24,7 \\
Orbicular & 77 & 10,9 \\
Temporal & 7 & 1,0 \\
Zigoma & 256 & 36,0 \\
Total & 711 & 100 \\
\hline
\end{tabular}

sendo descrita em 444 pacientes, seguida pelas queimaduras, relatadas em 315 pacientes (Figura 2).

As lesões de partes moles estavam presentes em $75,1 \%$ dos pacientes enquanto as fraturas foram encontradas em $24,9 \%$ dos pacientes. O nariz foi o local mais acometido nas fraturas de face $(76,8 \%)$, seguido pela fratura do zigoma $(9,6 \%)$. Quanto à causa das fraturas, a agressão física e os acidentes de automóveis/moto foram as principais causas em $48,0 \%$ e $15,8 \%$ dos pacientes, respectivamente (Tabela 4).
Os principais tratamentos instituídos foram: o curativo, a sutura e a redução de fratura (Tabela 5). A anestesia local foi realizada em $34 \%$ dos pacientes. O tratamento multidisciplinar foi instituído em $12 \%$ da população de estudo, sendo que as principais especialidades para encaminhamento foram a Oftalmologia $(65,7 \%)$, seguida pela Neurocirurgia $(17,6 \%)$ e pela Odontologia $(8,3 \%)$.

\section{DISCUSSÃO}

A maioria dos pacientes com trauma de face atendidos na Unidade de Emergência do Serviço de Cirurgia Plástica do HRAN foram do sexo masculino $(72,8 \%)$, semelhante a outros estudos ${ }^{9-11}$. Entretanto, existe uma tendência mundial ao aumento da incidência nas mulheres que estão cada vez mais expostas aos fatores de risco deste tipo de trauma ${ }^{12}$. O maior envolvimento na prática de atividades físicas, o maior número de mulheres motoristas, o aumento da violência das cidades associado à maior participação das mulheres em atividades extra-domiciliares, as aproximam do grupo de risco dos homens.

Os pacientes entre 21 a 30 anos constituíram a faixa etária de maior incidência entre as vítimas de trauma de face, achado este consistente com diversos estudos ${ }^{10,11,13,14}$.

Há três décadas os estudos apontavam os acidentes por veículos automotores como a principal causa dos traumas

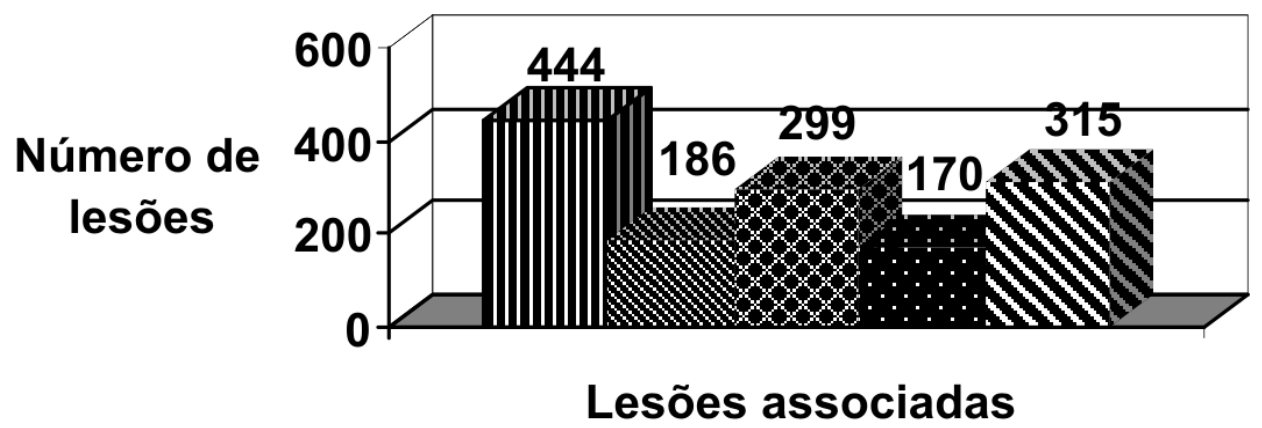

II Contusão e escoriação * Fratura

Lesão Cortocontusas

- Perda de Substância

Q Queimadura

Figura 2 - Tipo de lesões dos pacientes com trauma de face. 
Tabela 4 - Pacientes com fratura facial, segundo a localização da lesão e a causa, atendidos no Hospital Regional da Asa Norte, Brasília, DF, de janeiro a dezembro de 2004.

\begin{tabular}{|c|c|c|c|c|c|c|c|c|}
\hline \multicolumn{9}{|c|}{ Causas } \\
\hline Localização & AVM & $\mathbf{A F}$ & QB & $\mathbf{E}$ & INRQ & QPA & $\mathbf{A}$ & Total \\
\hline Frontal & & & 1 & & & 1 & & 2 \\
\hline Mandibular & 1 & & 3 & & 1 & 1 & & 6 \\
\hline Nasal & 21 & 70 & 10 & 13 & 10 & 11 & 1 & 136 \\
\hline Orbicular & 2 & 10 & 1 & & 2 & 1 & & 16 \\
\hline Zigoma & 4 & 5 & 1 & 1 & 4 & 2 & & 17 \\
\hline Total & 28 & 85 & 16 & 14 & 17 & 16 & 1 & 177 \\
\hline
\end{tabular}

Acidente veículo/moto $=A V M$, Agressão física $=A F$, Queda de bicicleta $=Q B$, Esporte $=E$, Impacto não relacionado à queda $=I N R Q, Q u e d a$ da própria altura $=Q P A$, Atropelamento $=A$

Tabela 5 - Pacientes com trauma facial, segundo o tratamento principal instituído, atendidos no Hospital Regional da Asa Norte, Brasília, DF, de janeiro a dezembro de 2004.

\begin{tabular}{lrc}
\hline Tratamento da lesão & N & \% \\
\hline Curativo & 383 & 54,1 \\
Desbridamento & 7 & 1,0 \\
Drenagem & 3 & 0,38 \\
Reconstrução com rrrretalho/enxerto & 12 & 1,7 \\
Redução & 153 & 21,6 \\
Sutura simples & 153 & 21,6 \\
Total & 711 & 100 \\
\hline
\end{tabular}

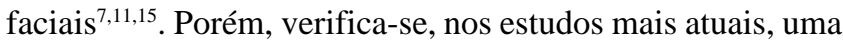
participação cada vez maior da agressão física como mecanismo de trauma facial devido o aumento da violência nas cidades ${ }^{12,16-19}$. Além disso, as políticas públicas buscando um maior controle e penalizações ao excesso de velocidade, o uso de cinto de segurança e o limite de velocidades nas vias são creditadas ao decréscimo do número de traumas faciais devido a acidentes com veículos automotores ${ }^{11}$. A introdução de dispositivos de segurança, como o uso obrigatório de cinto de segurança, "air bags", barras de proteção laterais e a proibição de dirigir alcoolizado não só diminuíram os índices de acidentes automobilísticos como causa de trauma facial, como a complexidade das fraturas faciais ${ }^{12,16,20}$. A adoção do cinto de segurança nos EUA, em um período de 10 anos, diminuiu a incidência de fraturas múltiplas faciais de $46,3 \%$ para $20,1 \%{ }^{21}$. Os estudos que não mostraram essa mudança, o motivo talvez seja a inclusão de pacientes vítimas de trauma das décadas de oitenta e noventa em que o uso dos equipamentos de proteção nos veículos não eram obrigatórios, principalmente no Brasil $^{11,14,20}$.

A maioria dos pacientes atendidos no hospital do estudo possui baixo poder aquisitivo entre outros problemas socioeconômicos. O aumento da violência urbana está associado aos conflitos socioeconômicos e emocionais aos quais muitas pessoas são submetidas, principalmente as mais jovens. A redução da violência interpessoal como causa de trau- ma de face será muito mais complicado e difícil que a redução do trauma de face decorrente de acidente automobilístico, pois a tendência da violência urbana e dos conflitos sociais é aumentar. Não se observaram, nos últimos anos, investimentos significativos do governo em segurança pública ou educação da população, enquanto isso as taxas de desemprego se mantêm elevadas.

A queda da própria altura mostrou-se como importante mecanismo de trauma nos extremos de idade, destacando-se em quase $73 \%$ das vítimas acima de 60 anos. O envelhecimento diminui gradativamente as funções biológicas, apresentando deficiências sensoriais múltiplas, comprometimento da cognição e da memória, associado ao uso de medicamentos pscicotrópicos e doenças osteomusculares aumentando o risco de queda ${ }^{11}$. Enquanto nas crianças, múltiplos fatores favorecem a queda, uma vez que a locomoção e o equilíbrio estão em desenvolvimento, além disso, desconhecem o limite de atos perigosos ${ }^{7,22}$.

O nariz é o principal local das fraturas de face, seguido pelo zigoma.Tal fato foi concordante com outros estu$\operatorname{dos}^{5,10,23}$. O motivo encontra-se no fato do nariz ocupar uma posição central na face e ser uma estrutura facilmente fraturada devido à pequena espessura dos ossos nasais. Além disso, foi verificado que a principal causa da fratura nasal é a agressão física.

Quanto às características das lesões faciais, observaram-se, principalmente, lesões corto-contusas ou queimaduras, cujos tratamentos resumiram-se apenas no curativo ou na sutura simples. Por outro lado, 23,3\% dos pacientes com trauma de face atendidos no Pronto-Socorro de Cirurgia Plástica necessitaram de procedimentos especializados, como redução de fratura facial e/ou reconstrução com retalho ou enxerto.

Diante dos achados encontrados, o paciente com trauma de face, atendido no Pronto-Socorro de Cirurgia Plástica, foi geralmente um jovem do sexo masculino, entre 21 e 30 anos e vítima de violência interpessoal. A queda da própria altura mostrou-se como importante mecanismo de trauma nos extremos de idade. As principais lesões foram queimaduras ou corto-contusas, tratadas geralmente com curativos ou sutura simples. 


\begin{abstract}
Background: To evaluate the etiology, age, gender and location of the lesions of facial trauma in patients arriving at our hospital. Methods: The data were evaluated through retrospective analysis of patient charts from January $1^{\text {st }}$ to December $31^{\text {st }}$, 2004. Results: The group comprised 711 patients with facial trauma. Blunt assault was the most common cause, followed by motor vehicle crashes. Falls were, by far, the predominant cause of injury in children, but with increasing age, assaults became more common. It was observed a male:female ratio of 3:1. The most frequent age group was the 21-30 years one, with $52 \%$. Facial fractures were found in 24.9\% of facial injuries. The most frequent fracture was nasal (76.8\%). Conclusion: Aggressions or interpersonal violence were the main cause of facial trauma in our population. The causes of facial trauma were directly related to the age.
\end{abstract}

Key words: Face; Maxillofacial Injuries; Facial Bones; Facial Injuries; Wounds and Injuries; Violence; Surgery.

\section{REFERÊNCIAS}

1. Krug EG, Sharma GK, Lozano R. The global burden of injuries. Am J Public Health. 2000;90(4):523-6.

2. Bisson JI, Shepherd JP, Dhutia M. Psychological sequelae of facial trauma. J Trauma. 1997;43(3):496-500.

3. Sastry SM, Sastry CM, Paul BK, Bain L, Champion HR. Leading causes of facial trauma in the major trauma outcome study. Plast Reconstr Surg. 1995;95(1):196-7.

4. Barker R, Hockey R, Spinks D, Miles E. Facial Injury. Injury Bulletin Queensland Injury Surveillance Unit. 2003;79:1-6.

5. Ong TK, Dudley M. Craniofacial trauma presenting at an adult accident and emergency department with an emphasis on soft tissue injuries. Injury. 1999;30(5):357-63.

6. Mackenzie EJ. Epidemiology of injuries: current trends and future challenges. Epidemiol Rev. 2000;22(1):112-9.

7. Wulkan M, Parreira Júnior JG, Botter DA. Epidemiologia do trauma facial. Rev Assoc Med Bras. 2005;51(5):290-5.D

8. Al-Qurainy IA, Stassen LF, Dutton GN, Dutton GN, Moos KF, el-Attar A. The characteristics of midfacial fractures and the association with ocular injury: a prospective study. Br J Oral Maxillofac Surg. 1991;29(5):291-301.

9. Zargar M, Khaji A, Karbaksh M, Zari MR. Epidemiology study of facial injuries during a 13 month of trauma registry in Tehran. Indian J Med Sci. 2004;58(3):109-14.

10. Sobreira T, Vieira JAO, Lobo AR, Wanderley JNB, Costa LJ. Prevalência de traumatismos bucomaxilofaciais em João Pessoa Brasil. Rev Bras Cien Saúde. 2002;6(1):25-32.

11. Montovani JC, Campos LMP, Gomes MA, Moraes VRS, Ferreira FD, Nogueira EA. Etiologia e incidência das fraturas faciais em adultos e crianças: experiência em 513 casos. Rev Bras Otorrinolaringol. 2006;72(2):235-41.

12. Beck RA, Blakeslee DB. The changing picture of facial fractures. 5-Year review. Arch Otolaryngol Head Neck Surg. 1989;115(7):826-9.

13. Marker P, Nielsen A, Bastian HL. Fractures of the mandibular condyle. Part 2: results of treatment of 348 patients. Br J Oral Maxillofac Surg. 2000;38(5):422-6.

14. Rodrigues FH, Miranda ES, Sousa VEM, Castro VM, Oliveira DRF, Leão CEG. Avaliação do trauma bucomaxilofacial no Hospital Maria Amélia Lins da Fundação Hospitalar do Estado de Minas Gerais. Rev Soc Bras Cir Plast. 2006; 21(4):211-6.
15. Luce EA, Tubb TD, Moore AM. Review of 1,000 major facial fractures and associated injuries. Plast Reconstr Surg. 1979;63(1):26-30.

16. Brook IM, Wood N. Aetiology and incidence of facial fractures in adults. Int J Oral Surg. 1983;12(5):293-8.

17. Magennis P, Shepherd J, Hutchison I, Brown A. Trends in facial injury: increasing violence more than compensates for decreasing road trauma [editorial]. BMJ. 1998;316(7128):325-6.

18. Scherer M, Sullivan WG, Smith DJ, Phillips LG, Robson MC. An analysis of 1,423 facial fractures in 788 patients at an urban trauma center. J Trauma. 1989;29(3):388-90.

19. Telfer MR, Jones GM, Shepherd JP. Trends in the aetiology of maxillofacial fractures in the United Kingdom (1977-1987). Br J Oral Maxillofac Surg. 1991; 29(4):250-5.

20. Shapiro AJ, Johnson RM, Miller SF, McCarthy MC. Facial fractures in a level I trauma centre: the importance of protective devices and alcohol abuse. Injury. 2001;32(5):353-6.

21. Convington DS, Wainwright DJ, Teichgraeber JF, Parks DH. Changing patterns in the epidemiology and treatment of zygoma fractures: 10-year review. J Trauma. 1994;37(2):243-8.

22. Shaikh ZS, Worrall SF. Epidemiology of facial trauma in a sample of patients aged 1-18 years. Injury. 2002;33(8):669-71.

23. Souza LC, Fischman R, Lucca ME, Vita Júnior J. Fratura dos ossos da face: análise de 455 casos. Rev Col Bras Cir. 1984;11(2):33-8.

Como citar este artigo:

Macedo JL, Camargo LM, Almeida PF, Rosa SC. Perfil epidemiológico do trauma de face dos pacientes atendidos no pronto socorro de um Hospital Público. Rev Col Bras Cir. [periódico na Internet] 2008; 35(1). Disponível em URL: http://www.scielo.br/rcbc

Endereço para correspondência:

Dr. Jefferson Lessa Soares de Macedo

SQS 213 Bloco "H" Apto 104 - Asa Sul

70.292-080 - Brasília-DF.

Telefone: (61) 99895746

Telefax: (61) 33278415

E-mail: jlsmacedo@yahoo.com.br 